\title{
El hábitat de los ríos mediterráneos. Diseño de un índice de diversidad de hábitat.
}

\author{
Isabel Pardo ${ }^{1}$, Maruxa Álvarez ${ }^{1}$, Jesús Casas ${ }^{2}$, José Luis Moreno ${ }^{3}$, Soledad Vivas ${ }^{2}$,
} Núria Bonada ${ }^{4}$, Javier Alba-Tercedor ${ }^{5}$, Pablo Jáimez-Cuéllar ${ }^{5}$, Gabriel Moyà ${ }^{6}$, Narcís Prat ${ }^{4}$, Santiago Robles ${ }^{7}, \mathrm{M}^{\mathrm{a}}$ Luisa Suárez ${ }^{3}$, Manuel Toro ${ }^{7}$ y M$^{\mathrm{a}}$ Rosario Vidal-Abarca ${ }^{3}$

${ }^{1}$ Área de Ecología. Universidad de Vigo. Campus Lagoas-Marcosende. 36200 Vigo.

${ }^{2}$ Departamento de Biología Vegetal y Ecología. Universidad de Almería. Cañada de San Urbano, s/n. 04120 Almería.

${ }^{3}$ Departamento de Ecología e Hidrología. Universidad de Murcia. Campus de Espinardo. 30100 Murcia.

${ }^{4}$ Departament d'Ecologia. Universitat de Barcelona. Diagonal, 645. 08028 Barcelona.

${ }_{5}^{5}$ Departamento de Biología Animal y Ecología. Universidad de Granada. Campus Universitario de Fuentenueva. 18071 Granada.

${ }^{6}$ Departament de Biologia. Universitat de les Illes Balears. Crta. Valldemosa, km. 7.5. 07071 Palma de Mallorca.

${ }^{7}$ CEDEX. División de Ecología de los Sistemas Acuáticos Continentales. Paseo Bajo Virgen del Puerto, 3. 28005 Madrid.

\section{RESUMEN}

Los ríos mediterráneos han padecido alteraciones históricas en sus cuencas y riberas, y como consecuencia el hábitat fluvial se ha visto afectado. El índice de hábitat fluvial (IHF) surge como respuesta a la necesidad de caracterizar los cauces de los ríos mediterráneos dentro de los objetivos generales del proyecto coordinado GUADALMED. Este proyecto pretende valorar el estado ecológico de los ríos mediterráneos de acuerdo con la Directiva Marco del Agua (2000/60/EC), la nueva legislación comunitaria europea de la gestión del agua. El IHF valora aspectos físicos del cauce relacionados con la heterogeneidad de hábitats y que dependen en gran medida de la hidrología y del sustrato existente, como son la frecuencia de rápidos, la existencia de distintos regímenes de velocidad y profundidad, el grado de inclusión del sustrato y sedimentación en pozas, y la diversidad y representación de sustratos. También se evalúa la presencia y dominancia de distintos elementos de heterogeneidad, que contribuyen a incrementar la diversidad de hábitat físico y de las fuentes alimenticias, entre ellos materiales de origen alóctono (hojas, madera) y de origen autóctono, como la presencia de diversos grupos morfológicos de productores primarios. Se confirma la dependencia de la calidad biológica (índices biológicos y número de familias) de la calidad del hábitat (MGL $\mathrm{p}<0,0001$ ), incluso después de sustraer el efecto de las otras covariables relacionadas con los patrones generales de distribución de invertebrados en los ríos mediterráneos (conductividad eléctrica, caudal y contaminación). El índice presenta un alto potencial para valorar el grado de alteración del hábitat de los ríos mediterráneos, mediante comparación con valores del IHF existentes en localidades de referencia con muy buen estado ecológico, objetivos a desarrollar en la segunda fase del Proyecto.

Palabras clave: hábitat, ríos mediterráneos, España.

\begin{abstract}
Mediterranean rivers suffer from historical alterations in their watersheds and riparian corridors, and as a consequence their stream habitats are strongly impacted. The river habitat index (IHF) is designed as a response to the need for characterising the physical habitat of Mediterranean streams and rivers within the main objectives of the GUADALMED project. The aim of this project is to evaluate the ecological status of Mediterranean rivers in accordance with the Water Framework Directive (2000/60/EC), the new European Community legislation on water management. The IHF evaluates relations between habitat heterogeneity and physical variables of the stream channel, which are influenced by hydrology and substrate composition. This includes variables such as frequency of riffles, flow velocity and depth regime, substrate diversity and substrate inclusion in riffles and sedimentation in pools. The index also evaluates presence and dominance of heterogeneity
\end{abstract}


elements, which contribute to increasing physical habitat diversity and food sources, among these allochthonous material (leaves, wood), and autochthonous primary producers as presence and dominance of different morphological groups of aquatic vegetation. This study identifies the strong dependence of the biological quality (biotic indices and number of families) on habitat quality (GLM p <0,0001), after elimination of the influence of other co-variables related to general patterns of invertebrate distribution in the studied Mediterranean streams (conductivity, discharge and contamination). The index has good potential for the evaluation of degree of disturbance in Mediterranean rivers through comparison of IHF values characteristic of reference conditions of high ecological status, in line with the objectives, which will be further developed in the following phase of the Project.

Key words: habitat, Mediterranean rivers, Spain.

\section{INTRODUCCIÓN}

El índice de hábitat fluvial (IHF) pretende valorar la capacidad del hábitat físico para albergar una fauna determinada. A una mayor heterogeneidad y diversidad de estructuras físicas del hábitat le corresponde una mayor diversidad de las comunidades biológicas que lo ocupan (Smith \& Smith, 2000). El hábitat suministra espacio físico y proporciona fuente de alimento para las especies. Estas características del hábitat constituyen The Templet en el cual la evolución forja estrategias de vida características que adaptan a las especies al ambiente (Southwood, 1988). La heterogeneidad del hábitat fluvial se considera actualmente como uno de los principales factores de influencia de la riqueza de especies de invertebrados acuáticos (Voelz \& McArthur, 2000). Bajo esta premisa se han desarrollado técnicas y métodos de muestreo en ríos para evaluar la calidad biológica en función de los macroinvertebrados, y en los cuales se contempla el muestreo de todos los hábitats fluviales existentes para obtener listados exhaustivos de las especies presentes (Wright et al., 1984; Davies, 1994; Barbour et al., 1999).

El desarrollo del índice de hábitat fluvial (IHF), aplicado en el proyecto GUADALMED, está basado inicialmente en las características evaluadas en el RHS (River Habitat Survey), el protocolo de muestreo de hábitats fluviales desarrollado en el Reino Unido (National Rivers Authority, 1995), y utilizado parcialmente en la elaboración de un índice de hábitat fluvial para un río gallego (Pardo et al., 1998). Este índice fue utilizado parcialmente junto con modifica- ciones durante el ejercicio de ínter calibración en Murcia (Bonada et al., (a) en este volumen), aunque sin resultados satisfactorios. Se decidió entonces modificarlo y completarlo con nuevas variables del hábitat generadas dentro de los grupos de investigación del proyecto GUADALMED, y con otras variables de relevancia usadas en la evaluación del hábitat fluvial para ríos de América del Norte (Barbour et al., 1999), que parecían tener más importancia en los ríos mediterráneos estudiados.

El IHF valora aspectos físicos del cauce relacionados con la heterogeneidad de hábitats y que dependen en gran medida de la hidrología y del sustrato existente. Entre ellos, la frecuencia de rápidos, la existencia de distintos regímenes de velocidad y profundidad, el grado de inclusión y sedimentación en pozas, y la diversidad y representación de sustratos. También se evalúa la presencia y dominancia de distintos elementos de heterogeneidad, que contribuyen a incrementar la diversidad de hábitat físico y de las fuentes alimenticias, entre ellos materiales de origen alóctono (hojas, madera) y de origen autóctono, como la presencia de diversos grupos morfológicos de productores primarios. Estos elementos alóctonos provienen mayoritariamente de la vegetación de ribera y contribuyen energéticamente al funcionamiento de estos sistemas aportando materia orgánica (hojas, madera, frutos,...) (Hynes, 1970; Fisher \& Likens, 1973), y limitando la entrada de luz a los cauces, condicionando así la existencia de gradientes ambientales de transición entre el río y la vegetación terrestre adyacente (Brosofske et al., 1997). La vegetación acuática autóctona de los ríos viene determinada 
por las condiciones de exposición a la luz, hidrología, nutrientes y la existencia de un sustrato apropiado (Fox, 1996). La alternancia y variación natural entre fuentes alóctonas y autóctonas de materia orgánica puede verse modificada por cambios en el uso del suelo, deforestación, urbanización, etc. Estos últimos cambios son susceptibles de alterar la hidrología superficial, la relación natural entre las fuentes alternativas de energía características de cada sistema fluvial y como consecuencia el hábitat físico.

El índice de hábitat fluvial surge de la necesidad de caracterizar los cauces de los ríos mediterráneos dentro de los objetivos generales del proyecto GUADALMED que pretende valorar el estado ecológico de los ríos Mediterráneos para adaptarse a la Directiva Marco del Agua (Directiva 2000/60/EC), donde se plantea la necesidad de evaluar la calidad de los distintos componentes estructurales hidromorfológicos de los ecosistemas fluviales. Los ríos mediterráneos han padecido históricas alteraciones de sus cuencas y riberas (Suárez et al., en este volumen), pudiendo verse visto afectado el hábitat fluvial. Este índice pretende valorar el grado de alteración del hábitat de ríos mediterráneos en su comparación con zonas de referencia de muy buen estado ecológico, y generación de rangos de calidad de hábitat en relación a otros parámetros biológicos utilizados en el proyecto GUADALMED para evaluar el estado ecológico del agua.

\section{MATERIAL Y MÉTODOS}

El IHF consta de siete bloques o apartados en los que se valora de manera independiente la presencia de distintos componentes en el cauce fluvial. La puntuación final del índice es el resultado de la suma de la puntuación obtenida en cada uno de los bloques y nunca puede ser mayor que 100. El estadillo para el cómputo del IHF se presenta en el Apéndice 1. Se suministran indicaciones adicionales para su estima en el campo en el capítulo del protocolo del proyecto GUADALMED (Jáimez-Cuéllar et al., en este volumen).
Las puntuaciones del índice de hábitat analizadas en este trabajo corresponden a la evaluación realizada por cada uno de los grupos del proyecto GUADALMED durante las campañas de muestreo del año 2000-2001. La matriz del IHF consta de 465 muestras, recogidas en 156 localidades, y para cada una se analizan los 7 componentes del índice y la puntuación final. Para explorar y analizar las relaciones entre el IHF y los restantes parámetros biológicos utilizados en la caracterización ecológica de los ríos mediterráneos, índices bióticos (IBMWP, IASPT, Alba-Tercedor y Sánchez-Ortega, 1988), riqueza de familias, índice de ribera QBR (Munné et al., 1998), y físico-química, se utilizó la matriz anterior sin las muestras de las cuencas del Júcar y Turia, ya que a la fecha de publicación no se encontraba finalizado el análisis de los datos biológicos para el 2000, resultando una matriz con 280 muestras. Los valores del QBR utilizados corresponden al valor registrado en cada localidad en el año 1999.

Para el análisis de los resultados se han utilizado las técnicas de análisis de componentes principales (ACP), Modelo General Lineal (MLG), regresión logística y coeficientes de correlación de Spearman, todas ellas ejecutadas con el programa estadístico SPSS versión 10.1.

De forma previa al análisis de los datos en este estudio se han realizado unas últimas modificaciones en el IHF. Variando la asignación de una puntuación de un apartado a otro (regímenes de velocidad / profundidad), y nuevas puntuaciones para la circunstancia de que existan "Sólo pozas" y la valoración de la sedimentación de las mismas. Estas modificaciones valoran el caso particular de los ríos temporales donde al final del periodo del agua sólo quedan pozas, muy heterogéneas y diversas.

\section{RESULTADOS}

\section{EI IHF y sus componentes}

Los datos del IHF presentan una distribución de frecuencias normal, con un rango comprendido 


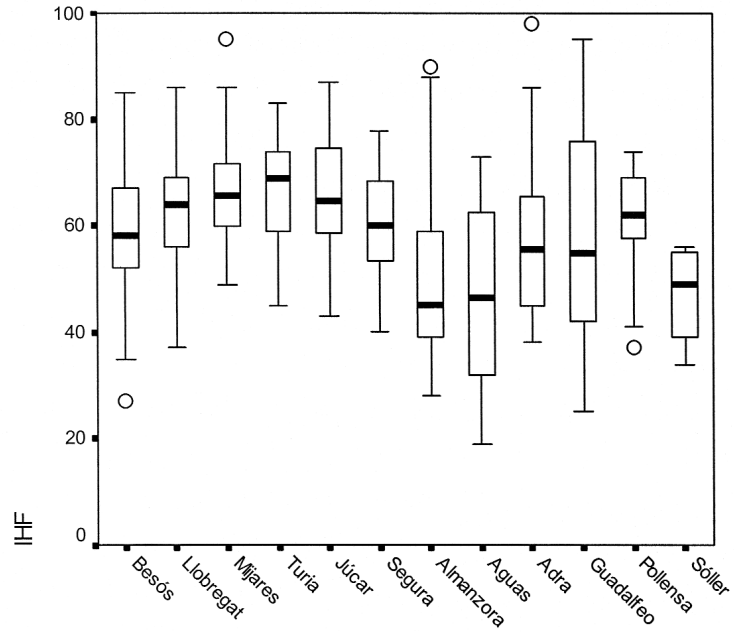

Figura 1. Diagrama de cajas de los valores del IHF registrado por los grupos componentes del proyecto GUADALMED. AL, Almería; CA, Cataluña; CED, CEDEX (Madrid); GR, Granada; IB, Islas Baleares; MU, Murcia. Box plots of IHF values per research group within the GUADALMED project. AL, Almería; CA, Cataluña; CED, CEDEX (Madrid); GR, Granada; IB, Islas Baleares; MU, Murcia.

entre 19 y 98, y un valor medio de 61 para el total de 465 casos. En la figura 1 se resumen los valores del IHF por cuenca fluvial estudiada en el proyecto GUADALMED.

Para evaluar la relación entre los distintos componentes del IHF se realizó un análisis de componentes principales sobre la matriz de 465 muestras. El primer eje explicó el 37.6\% de la varianza, y el segundo eje el 16.2\%. El eje I viene determinado en su parte positiva por todos los componentes del índice, pero principalmente por la frecuencia de rápidos, los regímenes de velocidad / profundidad y los elementos de heterogeneidad (Fig. 2). El eje II muestra el porcentaje de sombra en el cauce y los elementos de heterogeneidad asociados a su extremo negativo $\mathrm{y}$ enfrentados a la diversidad de sustrato mineral en el positivo. El coeficiente de correlación de Spearman entre los valores del IHF (no incluido en el análisis) y los valores del eje I del ACP, fue de $0.92(\mathrm{p}<0.01)$, indicando que este eje se puede identificar con el índice de hábitat.

La ordenación de las muestras del ACP anterior (Fig. 3) se representa como el valor medio

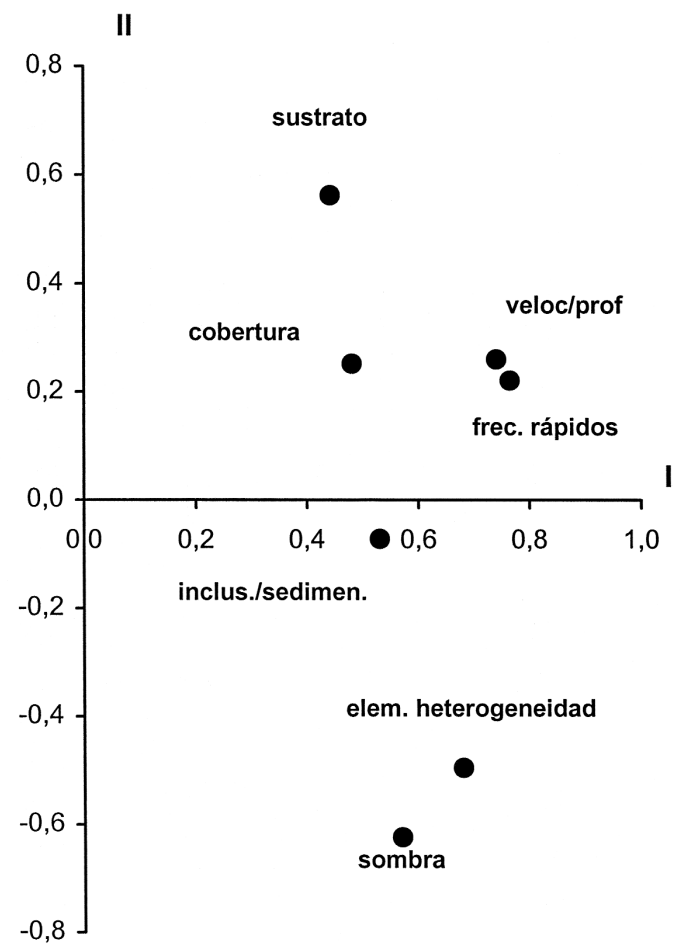

Figura 2. Ordenación (análisis de componentes principales) de los componentes del IHF. Ordination (principal component analysis) of IHF components.

de las localidades agrupadas por tipo de río de la tipología B modificada por expertos (Bonada et al., (b), en este volumen). Los tipos ordenados en la parte positiva del eje I tienen una media del IHF de 70, frente al valor medio de 48 para los tipos del extremo negativo del eje I. Para el eje II, el valor medio de los tipos en el extremo positivo es de 60 , y en el negativo de 62. Los tipos localizados en los extremos positivos de ambos ejes presentan valores altos del índice relacionados con una mayor diversidad de tamaños de sustrato mineral, mayor cobertura de vegetación acuática y mayor inclusión del sedimento, correspondientes a los ríos grandes y medios de zonas bajas calcáreos (tipos 4 y 5). Sin embargo, los valores más elevados del índice corresponden a los tipos localizados en el plano definido por las partes positiva del eje I y negativa del eje II, asociados a mayores puntuaciones del porcentaje de sombra en el cauce y 


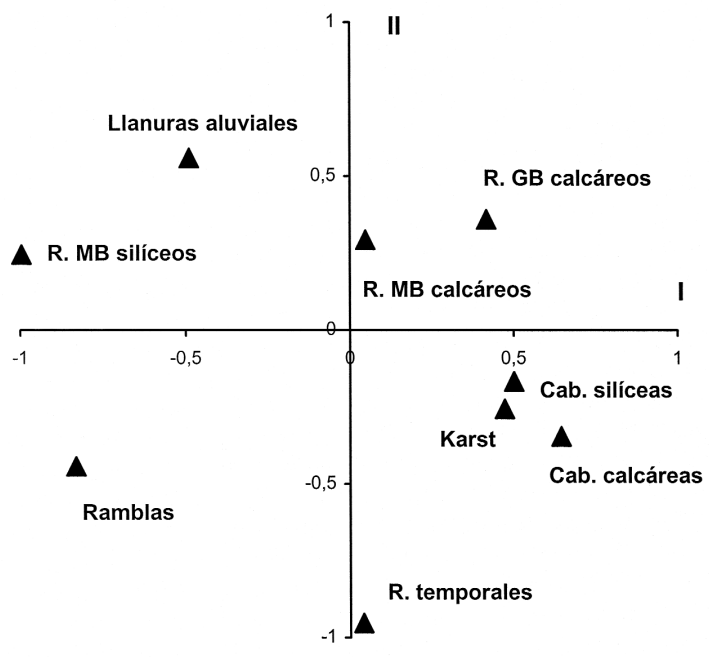

Figura 3. Ordenación (análisis de componentes principales) de las coordenadas medias por tipo de río (tipología B- modificado por la opinión de los expertos). MB, medio bajo; GB, grande bajo. Ordination (principal component analysis) of mean coordinates of river type (Tipology B-modified by the expert panel). $M B$, lowland midsize; $G B$, lowland large.

elementos de heterogeneidad, que incluyen mayoritariamente a las cabeceras calcáreas y silíceas y karst de distintas regiones (Fig. 3).

El valor del IHF se correlaciona en primer lugar con la cobertura vegetal en el cauce (coeficiente de correlación de Spearman, $r=0.70$, $\mathrm{p}=0.01)$, probablemente por ser el apartado con mayor puntuación en el mismo, seguido por el grado de inclusión / sedimentación $(r=0.62$, $\mathrm{p}=0.01)$, los elementos de heterogeneidad $(r=0.60, \mathrm{p}=0.01)$, la frecuencia de rápidos $(r=0.58, \mathrm{p}=0.01)$, y los regímenes de velocidad y profundidad $(r=0.57, \mathrm{p}=0.01)$.

\section{Relación entre el IHF y otros indicadores del estado ecológico de los ríos mediterráneos}

En un primer análisis se constató la existencia de correlaciones significativas (coeficiente de correlación de Spearman) entre el IHF y todos los parámetros biológicos considerados en este estudio. La más alta se da entre el IBMWP y el número de familias $(r=0.96, \mathrm{p}=0.01)$, y el IHF se correlacionó en mayor medida con el IBMWP $(r=0.63, \mathrm{p}=0.01)$, el IASPT $(r=0.52, \mathrm{p}=0.01)$, y el número de familias $(r=0.44, \mathrm{p}=0.01)$.

La existencia de relaciones positivas entre el IHF y los índices biológicos, IBMWP, IASPT y el número de familias, indica una dependencia que hay que explorar. Se realizaron análisis MLG utilizando como variables dependientes el IBMWP, IASPT y el número de familias, como variable independiente el IHF, y como covariables la conductividad eléctrica, contaminación (la interacción entre amonio y fosfatos) y caudal. Los modelos fueron significativos, indicando la existencia de relaciones de dependencia funcional de la biota con el hábitat que habita (Tabla 1, Fig. 4), incluso después de sustraer el efecto de las otras covariables relacionadas con

Tabla 1. Análisis de la varianza (MGL) de los índices bióticos y número de familias según el IHF, utilizando como covariables la interacción del amonio-fosfatos, caudal y conductividad eléctrica. Analyses of variance (GLM) of biotic index and number of families according to the IHF index. The ammonium-phosphate interaction, discharge and electric conductivity were used as covariates.

\begin{tabular}{lclrlrl}
\hline & IBMWP & \multicolumn{3}{c}{ IASPT } & \multicolumn{3}{c}{ SFAM } \\
\hline & \multicolumn{1}{c}{ F } & p & F & p & F & p \\
\hline Modelo corregido & 4.0 & 0.0000 & 3.8 & 0.0000 & 3.0 & 0.0000 \\
IHF & 3.3 & 0.0000 & 2.6 & 0.0000 & 2.5 & 0.0000 \\
AMONIO * FOSFATOS & 14.6 & 0.0002 & 34.6 & 0.0000 & 16.3 & 0.0001 \\
CAUDAL & 5.2 & 0.0243 & 11.4 & 0.0009 & 5.2 & 0.0239 \\
COND & 7.4 & 0.0072 & 6.5 & 0.0114 & 1.3 & 0.2506 \\
\hline
\end{tabular}

$r^{2}=0.58$

$r^{2}=0.58$

$r^{2}=052$ 

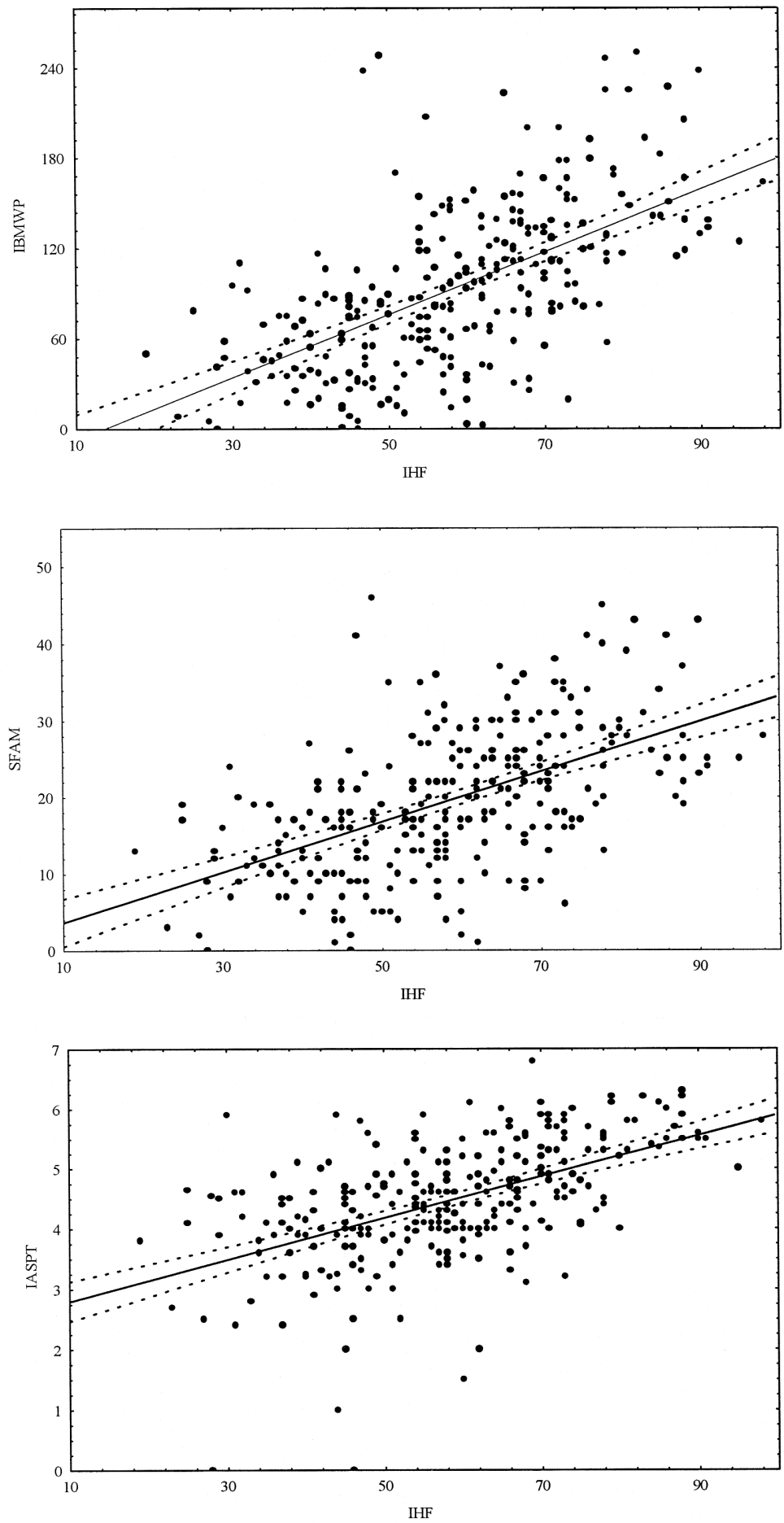

Figura 4. Relación entre los índices bióticos (IBMWP, IASPT) y el número de familias (SFAM), con el hábitat fluvial (IHF). Relationship between biotic index (IBMWP, IASPT) and number of families (SFAM), and the river habitat index (IHF). 
los patrones generales de distribución de invertebrados en los ríos mediterráneos, como son la salinidad, caudal y contaminación (Vivas et al., en este volumen), esta última expresada en los modelos como la interacción entre los valores de amonio y fosfato (Tabla 1).

En la figura 4 se ilustran estas relaciones, y se representan los intervalos de confianza para la línea de regresión para las variables biológicas. En ellas se hace evidente la importancia de la alteración del hábitat sobre las comunidades de invertebrados acuáticos, ya que la disminución de los valores del IHF se corresponden con un gradiente de disminución de la calidad biológica. En la figura se observa el potencial real que presenta el hábitat fluvial, evaluado con el IHF, para alojar una comunidad de invertebrados diversa. En términos generales, se observa que valores del IHF bajos (30 - 45) se corresponden con una calidad biológica de hasta 120 , y un número de familias presentes máximo de 26 . Los valores intermedios $(50$ - 70) pueden sostener una fauna de entre 19 y 37 familias y calidad biológica entre 80 y 220 . Por último los valores comprendidos entre 70 y 90 , pueden corresponder a una calidad biológica superior a $120 \mathrm{y}$ de hasta 250 , y una fauna de alta riqueza de familias, entre 25 y 45 . Valores del IHF por encima de 90 son escasos, bien por que no existan los máximos teóricos del hábitat óptimo, bien por que las localidades evaluadas no lleguen a él.

\section{Análisis de los grupos de la tipología. Localidades de referencia y no referencia}

En el proyecto GUADALMED se han estudiado distintas cuencas fluviales en la franja litoral mediterránea e Islas Baleares (Robles et al., en este volumen). Inicialmente se seleccionaron en las cuencas localidades representativas de los distintos tipos de ríos de la zona, y localidades consideradas de referencia de muy buen estado, según los expertos involucrados en los proyectos. Posteriormente se hizo un análisis de las localidades de referencia así seleccionadas, y se buscó su conformidad con los criterios actuales sobre lo que se interpreta como condiciones de referencia y ausencia de presiones antropogénicas (Bonada et al., (c) en este volumen). Los criterios de corte en ese análisis fueron: valor $>100$ para el IBMWP y $>75$ para el QBR. Así de las 54 localidades seleccionadas inicialmente como de referencia, tan sólo 15 cumplen con estos criterios utilizados al $100 \%$. Sin embargo estos cortes, ajustados a las clases de calidad de dichos índices, pueden ser demasiado estrictos o permisibles, para determinados tipos de ríos dentro del conjunto de los ríos mediterráneos. Por ejemplo, ríos localizados en zonas áridas y semiáridas de la Península pueden no presentar el potencial adecuado para el asentamiento de una vegetación de ribera arbórea (Suárez et al., en este volumen), por lo tanto utilizar un corte del QBR $>75$, puede ser un criterio imposible de alcanzar para algunos tipos de río mediterráneos. A la inversa, un valor del IBMWP cercano a 100 , puede ser muy bajo para algún tipo de río y estar indicando que son evidentes algunos signos de alteración.

En este trabajo se establece la dependencia entre las comunidades de invertebrados acuáticos y la calidad del hábitat fluvial (IHF), con independencia de la contaminación del agua, caudal y conductividad eléctrica. Por lo tanto, el hábitat por si mismo influencia de forma significativa a la comunidad de invertebrados en los ríos mediterráneos, y se constituye como criterio válido y a la vez complementario para establecer condiciones de referencia del muy buen estado ecológico para la biota.

A continuación se propone un ensayo metodológico que considera la inclusión del IHF como otro criterio de corte que añadir al IBMWP y QBR para establecer localidades de referencia, y se analiza posteriormente la posible mejora o no de su inclusión sobre las otras variables bióticas y físico-químicas. Para establecer un criterio de corte del IHF en condiciones de referencia, se analizaron en primer lugar los valores del mismo correspondiente a las 15 localidades de referencia establecidas en el proyecto GUADALMED (Bonada et al., (c)Anexo 2, en este volumen). El percentil 25 de los valores del IHF de las 15 localidades de 
Tabla 2. Listado de las 15 localidades de referencia seleccionada según Bonada et al., (b, en este volumen, Anexo 2) en la primera columna, al que se han añadido 12 localidades de referencia más según el criterio del IHF $>66$ y IBMWP $>100$, en la segunda columna. List of 15 reference sites according to Bonada et al., (b, in this volume, Annex 2) in the first column. 12 reference sites have been added with the criteria IHF $>66$ and IBMWP>100, in the second column.

\begin{tabular}{|c|c|c|c|c|c|}
\hline \multicolumn{3}{|c|}{ REFERENCIA } & \multicolumn{3}{|c|}{ REFERENCIA } \\
\hline $\begin{array}{l}\text { Grupo } \\
\text { tipología }\end{array}$ & & $\begin{array}{l}\text { Cumplen } \\
\text { criterios }\end{array}$ & SCR & $\begin{array}{l}\text { Podrían cumplirlos } \\
\text { fácilmente }\end{array}$ & SCR \\
\hline \multirow[t]{4}{*}{1} & $\mathrm{Sil} / \mathrm{Cab}$ & AD5-R & 10 & B32 & 9 \\
\hline & & B35-R & 10 & GU14-R & 7 \\
\hline & & GU11 & 10 & & \\
\hline & & GUl-R & 10 & & \\
\hline 3 & Sil/MB & & & & \\
\hline \multirow[t]{3}{*}{2} & Cal/Cab & L44-R & 10 & GU2-R & 9 \\
\hline & & SE1-R & 10 & L56-R & 7 \\
\hline & & SE2-R & 10 & SE3-R & 9 \\
\hline \multirow[t]{2}{*}{7} & Temporales & B24-R & 10 & B7a-R & 9 \\
\hline & & & & B7-R & 8 \\
\hline \multirow[t]{6}{*}{8} & Karst & L45-R & 10 & AL6-R & 7 \\
\hline & & PO9-R & 10 & AL7-R & 8 \\
\hline & & POl1 & 10 & AGl-R & 7 \\
\hline & & PO1 -R & 10 & AG2-R & 7 \\
\hline & & PO7-R & 10 & & \\
\hline & & PO8-R & 10 & & \\
\hline 4 & Cal/MB & L54 & 10 & SE5-R & 8 \\
\hline 5 & Cal/GB & & & & \\
\hline 6 & LlanAluvial & & & & \\
\hline 9 & Ramblas & & & & \\
\hline
\end{tabular}

referencia $(n=42)$ fue de 66 y la mediana de 68. Se utilizó el corte de 66 por tanto para definir el valor mínimo que una localidad de referencia debería presentar. Posteriormente, del listado de localidades que podrían fácilmente cumplir con los criterios de referencia (Bonada et al. (b)), se seleccionaron las que presentaban un valor del IHF $>66$ y un IBMWP $>100$; bien en una de las tres fechas del muestreo (sólo tres localidades), bien en más de una fecha de muestreo (9 localidades). Con estos criterios, se seleccionaron e incluyeron 12 localidades más al listado de 15 localidades de referencia inicial. Esta adición de 12 localidades $(n=78)$, aumenta la mediana del IHF a 71, y el valor del percentil 25 sigue en 66. En la Tabla 2 se presenta el listado de las 15 localidades de referencia más las 12 nuevas. Dentro de las nuevas localidades los grupos que más incrementan son los karsts (4 localidades) y las cabeceras calcáreas (3 localidades). Según esta aproximación, el porcentaje de localidades de referencia iniciales evaluadas por expertos que realmente cumplen con los criterios expuestos en esta publicación y en Bonada et al. (c, en este volumen), ascendería al $50 \%$.

En la figura 5 (inferior) se comparan los valores del IHF calculados sobre las 27 localidades de referencia $(\mathrm{R})$ y no referencia $(\mathrm{NR})$ para los grupos de la tipología B - modificados por expertos. De forma general las localidades de $\mathrm{R}$ (en los grupos donde las hay), presentan mayores puntuaciones del IHF frente a las NR (Fig. 5) (matriz con 280 casos). Las medianas más altas corresponden a las cabeceras silíceas (IHF de 74), seguido por los karsts (71), seguidos por las cabeceras y ríos medianos calcáreos y temporales con valores similares (68-66). Idéntico análisis de los valores del IHF con sólo las 

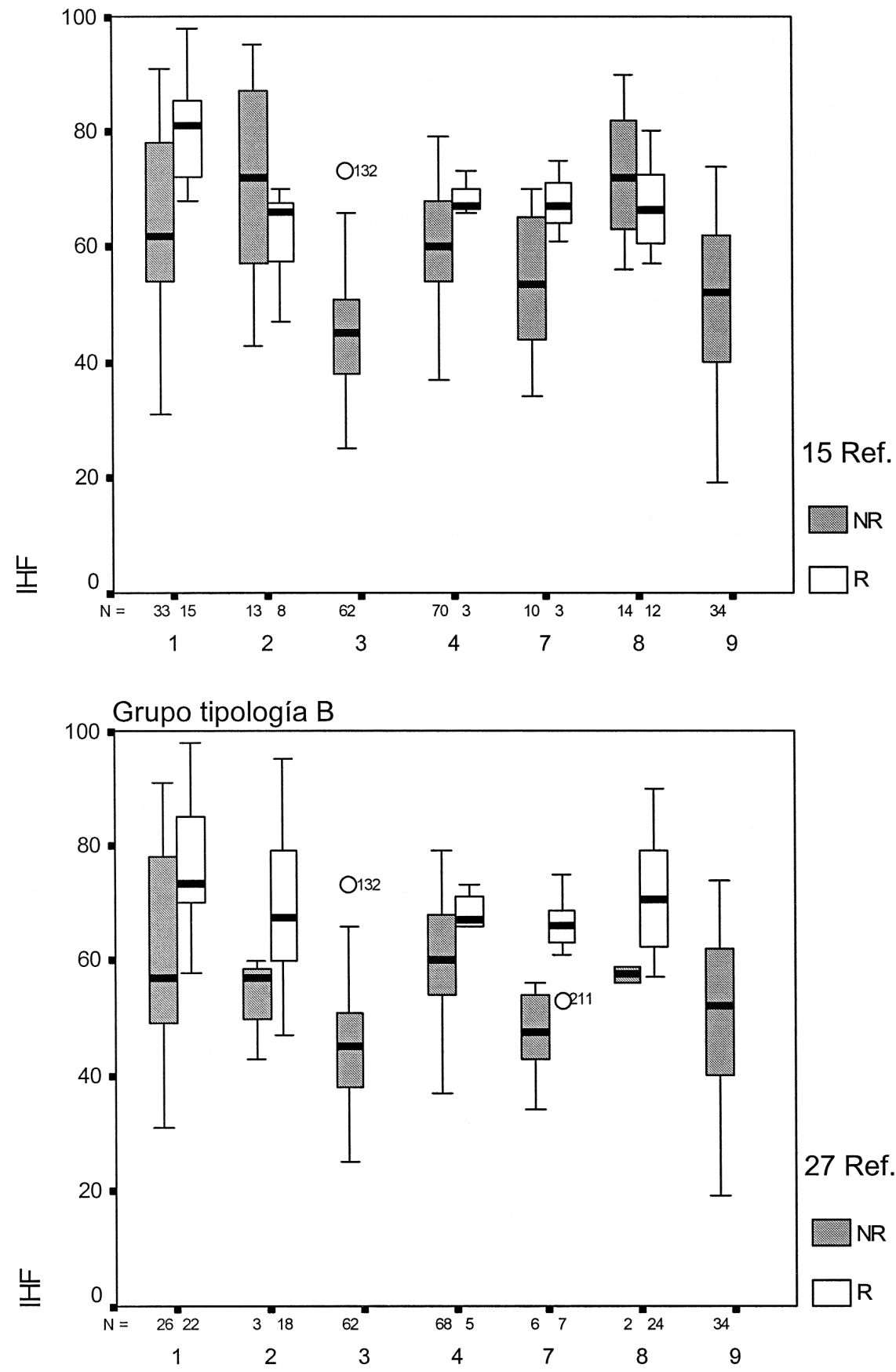

Grupo tipología B

Figura 5. Diagramas de cajas de los valores del IHF por tipo de río y para las localidades de referencia (R) y no referencia (NR). Se utilizan los valores estacionales de 15 localidades (gráfico superior) y 27 localidades (inferior) de referencia. Box plots of IHF values per river type and for reference $(R)$ and non reference (NR) sites. Seasonal values from 15 (upper graph) and 27 (lower graph) reference sites were used. 
Tabla 3. Mediana, percentil 25 , media y error estándar de los parámetros biológicos, y de los elementos hidromorfológicos y físico-químicos, de relevancia para la biota, para los grupos de la tipología B- modificada por expertos, y considerando los valores para los grupos de las muestras de 15 y 27 localidades de referencia. Median, 25 percentile, mean and standard error of biological parameters, and of hydro-morphological and physico-chemical variables of relevance for the biota, for groups of typology B-modified by the expert panel. Values for sample groups of 15 and 27 reference sites were considered.

\begin{tabular}{|c|c|c|c|c|c|c|c|c|c|c|c|c|c|c|c|c|}
\hline \multicolumn{10}{|c|}{27 referencias } & \multicolumn{7}{|c|}{15 referencias } \\
\hline & & \multicolumn{4}{|c|}{ NO REFERENCIA } & \multicolumn{4}{|c|}{ REFERENCIA } & \multicolumn{4}{|c|}{ NO REFERENCIA } & \multicolumn{3}{|c|}{ REFERENCIA } \\
\hline \multicolumn{2}{|c|}{ Tipo río } & Mediana & P25 & Media & $\mathbf{E E}$ & Mediana & P25 & Media & $\mathbf{E E}$ & Mediana & P25 & Media & $\mathbf{E E}$ & Mediana & P25 & Media \\
\hline \multirow[t]{8}{*}{1} & IHF & 57.0 & 48.3 & 61.0 & 3.5 & 73.5 & 69.8 & 76.8 & 2.0 & 62.0 & 51.5 & 63.1 & 2.9 & 81.0 & 71.0 & 79.5 \\
\hline & IBMWP & 103.5 & 63.8 & 97.0 & 7.7 & 131.5 & 107.8 & 129.4 & 6.4 & 106.0 & 75.0 & 100.1 & 6.5 & 138.0 & 112.0 & 137.5 \\
\hline & NFAM & 20.5 & 14.5 & 19.1 & 1.3 & 23.5 & 17.5 & 23.0 & 1.2 & 21.0 & 15.0 & 19.5 & 1.1 & 24.0 & 21.0 & 23.9 \\
\hline & IASPT & 4.9 & 4.6 & 4.9 & 0.1 & 5.7 & 5.3 & 5.7 & 0.1 & 5.1 & 4.6 & 5.0 & 0.1 & 5.8 & 5.4 & 5.8 \\
\hline & QBR & 70.0 & 48.8 & 63.3 & 4.4 & 87.5 & 80.0 & 85.7 & 2.0 & 70.0 & 50.0 & 68.6 & 4.0 & 85.0 & 70.0 & 84.3 \\
\hline & AMONIO & 0.0 & 0.0 & 0.1 & 0.0 & 0.0 & 0.0 & 0.2 & 0.1 & 0.1 & 0.0 & 0.1 & 0.0 & 0.0 & 0.0 & 0.2 \\
\hline & $\mathrm{CE}$ & 172.5 & 118.6 & 311.5 & 64.4 & 147.5 & 48.9 & 189.6 & 37.3 & 170.0 & 132.5 & 304.8 & 52.5 & 64.0 & 34.0 & 147.4 \\
\hline & CAUDAL & 345.8 & 3.6 & 761.9 & 221.0 & 45.9 & 13.1 & 148.8 & 46.9 & 99.3 & 7.2 & 611.0 & 180.9 & 65.5 & 18.3 & 194.5 \\
\hline \multirow[t]{8}{*}{2} & IHF & 57.0 & 43.0 & 53.3 & 5.2 & 67.5 & 59.0 & 69.9 & 3.2 & 72.0 & 56.5 & 70.8 & 4.6 & 66.0 & 56.3 & 62.4 \\
\hline & IBMWP & 32.0 & 3.0 & 23.7 & 10.4 & 155.0 & 129.0 & 160.9 & 10.1 & 129.0 & 75.0 & 121.6 & 17.6 & 166.0 & 147.5 & 172.0 \\
\hline & NFAM & 9.0 & 2.0 & 7.0 & 2.5 & 30.0 & 25.0 & 29.2 & 1.6 & 25.0 & 14.5 & 22.4 & 2.9 & 31.0 & 29.5 & 31.7 \\
\hline & IASPT & 3.2 & 1.5 & 2.9 & 0.7 & 5.6 & 5.2 & 5.5 & 0.1 & 5.6 & 4.3 & 5.0 & 0.4 & 5.4 & 4.9 & 5.3 \\
\hline & QBR & 20.0 & 20.0 & 20.0 & 0.0 & 90.0 & 65.0 & 83.9 & 3.3 & 65.0 & 37.5 & 62.7 & 7.7 & 95.0 & 90.0 & 93.3 \\
\hline & AMONIO & 13.2 & 10.4 & 12.9 & 1.4 & 0.1 & 0.0 & 0.1 & 0.0 & 0.1 & 0.1 & 3.3 & 1.7 & 0.1 & 0.0 & 0.1 \\
\hline & $\mathrm{CE}$ & 2040.0 & 1610.0 & 2516.7 & 702.7 & 372.4 & 238.0 & 439.3 & 60.6 & 307.0 & 159.8 & 866.4 & 302.8 & 408.0 & 361.4 & 514.8 \\
\hline & CAUDAL & 53.0 & 28.0 & 54.7 & 15.9 & 359.0 & 40.0 & 1873.7 & 1308.3 & 99.6 & 42.4 & 266.0 & 87.4 & 530.4 & 31.0 & 3589.7 \\
\hline \multirow[t]{8}{*}{3} & IHF & 45.0 & 37.8 & 45.2 & 1.3 & & & & & & & & & & & \\
\hline & IBMWP & 47.0 & 30.8 & 53.9 & 4.3 & & & & & & & & & & & \\
\hline & NFAM & 12.0 & 9.0 & 13.0 & 0.9 & & & & & & & & & & & \\
\hline & IASPT & 3.9 & 3.4 & 3.8 & 0.1 & & & & & & & & & & & \\
\hline & QBR & 35.0 & 20.0 & 34.0 & 2.2 & & & & & & & & & & & \\
\hline & AMONIO & 0.1 & 0.1 & 1.7 & 0.5 & & & & & & & & & & & \\
\hline & $\mathrm{CE}$ & 1042.5 & 662.8 & 1203.6 & 95.9 & & & & & & & & & & & \\
\hline & CAUDAL & 109.8 & 7.1 & 690.4 & 178.0 & & & & & & & & & & & \\
\hline \multirow[t]{8}{*}{4} & IHF & 60.0 & 54.0 & 60.0 & 1.3 & 67.0 & 66.0 & 68.6 & 1.4 & 60.0 & 54.0 & 60.2 & 1.2 & 67.0 & 66.0 & 68.7 \\
\hline & IBMWP & 78.5 & 55.8 & 87.6 & 6.1 & 115.5 & 111.0 & 124.3 & 7.4 & 81.0 & 58.0 & 88.7 & 5.9 & 138.0 & 111.0 & 134.7 \\
\hline & NFAM & 17.0 & 12.3 & 18.9 & 1.1 & 24.5 & 22.0 & 24.0 & 1.2 & 18.0 & 13.0 & 19.1 & 1.1 & 25.0 & 19.0 & 24.0 \\
\hline & IASPT & 4.4 & 4.0 & 4.4 & 0.1 & 5.2 & 4.8 & 5.2 & 0.2 & 4.4 & 4.0 & 4.4 & 0.1 & 5.5 & 5.5 & 5.6 \\
\hline & QBR & 45.0 & 22.5 & 49.3 & 3.4 & 75.0 & 55.0 & 75.0 & 8.9 & 45.0 & 30.0 & 49.6 & 3.3 & 95.0 & 95.0 & 95.0 \\
\hline & AMONIO & 0.1 & 0.1 & 0.8 & 0.4 & 0.0 & 0.0 & 0.0 & 0.0 & 0.1 & 0.1 & 0.7 & 0.4 & 0.1 & 0.1 & 0.1 \\
\hline & $\mathrm{CE}$ & 721.0 & 540.3 & 974.6 & 84.5 & 414.3 & 336.8 & 437.8 & 45.1 & 676.0 & 539.0 & 955.9 & 81.6 & 340.0 & 327.0 & 343.3 \\
\hline & CAUDAL & 167.5 & 39.0 & 417.5 & 64.3 & 797.5 & 292.6 & 2077.1 & 1488.2 & 189.0 & 44.0 & 541.7 & 142.0 & 800.0 & 795.0 & 798.3 \\
\hline \multirow[t]{8}{*}{7} & IHF & 47.5 & 40.8 & 47.0 & 3.4 & 66.0 & 61.0 & 65.3 & 2.6 & 53.5 & 43.8 & 53.6 & 3.6 & 67.0 & 61.0 & 67.7 \\
\hline & IBMWP & 84.0 & 67.5 & 90.2 & 10.2 & 97.0 & 81.0 & 95.9 & 9.8 & 86.0 & 67.5 & 93.1 & 8.8 & 97.0 & 81.0 & 93.7 \\
\hline & NFAM & 19.0 & 16.8 & 19.8 & 1.7 & 20.0 & 17.0 & 18.9 & 2.0 & 19.0 & 16.8 & 19.0 & 1.6 & 20.0 & 17.0 & 20.3 \\
\hline & IASPT & 4.3 & 3.8 & 4.6 & 0.4 & 5.1 & 4.8 & 5.2 & 0.2 & 5.1 & 4.1 & 5.0 & 0.3 & 4.8 & 4.3 & 4.7 \\
\hline & QBR & 45.0 & 25.0 & 57.9 & 15.4 & 85.0 & 65.0 & 80.0 & 5.3 & 65.0 & 25.0 & 63.2 & 10.1 & 90.0 & 90.0 & 90.0 \\
\hline & AMONIO & 0.0 & 0.0 & 0.1 & 0.1 & 0.3 & 0.1 & 0.5 & 0.2 & 0.0 & 0.0 & 0.3 & 0.1 & 0.1 & 0.1 & 0.3 \\
\hline & $\mathrm{CE}$ & 679.5 & 240.0 & 600.4 & 131.1 & 417.0 & 312.0 & 456.6 & 54.3 & 389.2 & 300.0 & 507.9 & 90.2 & 598.0 & 581.0 & 604.0 \\
\hline & CAUDAL & 0.5 & 0.0 & 467.2 & 452.2 & 1.5 & 1.0 & 4.1 & 1.9 & 1.5 & 0.3 & 299.6 & 288.1 & 1.0 & 0.4 & 1.1 \\
\hline
\end{tabular}


Tabla 3. Continuación. Continuation.

\begin{tabular}{|c|c|c|c|c|c|c|c|c|c|c|c|c|c|c|c|c|}
\hline \multicolumn{10}{|c|}{27 referencias } & \multicolumn{7}{|c|}{15 referencias } \\
\hline & & \multicolumn{3}{|c|}{ NO REFERENCIA } & \multicolumn{5}{|c|}{ REFERENCIA } & \multicolumn{3}{|c|}{ NO REFERENCIA } & \multicolumn{4}{|c|}{ REFERENCIA } \\
\hline \multicolumn{2}{|c|}{ Tipo río } & Mediana & P25 & Media & $\mathbf{E E}$ & Mediana & P25 & Media & $\mathbf{E E}$ & Mediana & P25 & Media & $\mathbf{E E}$ & Mediana & P25 & Media \\
\hline \multirow[t]{8}{*}{8} & IHF & 57.5 & 56.0 & 57.5 & 1.5 & 70.5 & 62.3 & 71.2 & 2.0 & 72.0 & 62.8 & 73.3 & 3.0 & 66.5 & 59.8 & 66.4 \\
\hline & IBMWP & 111.0 & 107.0 & 111.0 & 4.0 & 148.5 & 122.3 & 158.6 & 9.9 & 153.5 & 119.5 & 173.2 & 14.8 & 139.0 & 115.3 & 133.7 \\
\hline & NFAM & 27.0 & 27.0 & 27.0 & 0.0 & 31.5 & 27.3 & 32.2 & 1.4 & 32.0 & 27.0 & 33.7 & 2.0 & 30.0 & 26.0 & 29.6 \\
\hline & IASPT & 4.1 & 4.0 & 4.1 & 0.1 & 4.8 & 4.5 & 4.8 & 0.1 & 5.0 & 4.6 & 5.0 & 0.2 & 4.6 & 4.3 & 4.5 \\
\hline & QBR & 80.0 & 80.0 & 80.0 & 0.0 & 92.5 & 68.8 & 82.7 & 4.2 & 90.0 & 76.3 & 86.4 & 3.5 & 90.0 & 50.0 & 77.9 \\
\hline & AMONIO & 0.1 & 0.1 & 0.1 & 0.0 & 0.1 & 0.0 & 0.1 & 0.0 & 0.1 & 0.1 & 0.1 & 0.0 & 0.0 & 0.0 & 0.0 \\
\hline & $\mathrm{CE}$ & 1430.0 & 1225.0 & 1430.0 & 205.0 & 767.5 & 487.0 & 1340.4 & 245.8 & 1430.0 & 459.3 & 1827.6 & 377.8 & 767.5 & 585.4 & 786.9 \\
\hline & CAUDAL & 0.9 & 0.0 & 0.9 & 0.9 & 11.0 & 1.2 & 19.4 & 4.7 & 23.0 & 12.5 & 29.6 & 6.7 & 1.4 & 0.9 & 4.5 \\
\hline \multirow[t]{8}{*}{9} & IHF & 52.0 & 40.0 & 50.6 & 2.6 & & & & & & & & & & & \\
\hline & IBMWP & 73.0 & 45.0 & 68.7 & 5.8 & & & & & & & & & & & \\
\hline & NFAM & 16.5 & 12.0 & 16.8 & 1.3 & & & & & & & & & & & \\
\hline & IASPT & 4.0 & 3.7 & 3.9 & 0.1 & & & & & & & & & & & \\
\hline & QBR & 60.0 & 40.0 & 51.9 & 3.8 & & & & & & & & & & & \\
\hline & AMONIO & 0.0 & 0.0 & 0.5 & 0.2 & & & & & & & & & & & \\
\hline & $\mathrm{CE}$ & 3895.0 & 2443.0 & 11858.8 & 3407.9 & & & & & & & & & & & \\
\hline & CAUDAL & 3.9 & 0.1 & 42.6 & 33.0 & & & & & & & & & & & \\
\hline
\end{tabular}

15 localidades de referencia (superior) establecidas con sólo los criterios del IBMWP y QBR, resulta en un escaso papel discriminatorio del IHF entre R y NR para las cabeceras calcáreas, ríos temporales y karsts, siendo alguna mediana de las localidades de R más bajas que las de NR.

En la Tabla 3 se presentan los valores de la mediana, percentil 25 , media y error estándar de los parámetros biológicos, y de los elementos hidromorfológicos y físico-químicos de relevancia

Tabla 4. Modelos para la predicción de condiciones de referencia y no referencia. Se han utilizado los datos de 15 y de 27 localidades de referencia. Models for the prediction of reference and non reference conditions. Data from 15 and 27 reference sites have been included in analyses.

\begin{tabular}{|c|c|c|c|}
\hline & Variable & $\operatorname{Exp}(B)$ & $\mathbf{p}$ \\
\hline \multicolumn{4}{|l|}{15 referencias } \\
\hline & QBR & 1.07 & 0.000 \\
\hline \multicolumn{4}{|l|}{27 referencias } \\
\hline & IHF & 1.05 & 0.009 \\
\hline & IBMWP & 1.01 & 0.032 \\
\hline & IASPT & 2.06 & 0.030 \\
\hline & QBR & 1.04 & 0.001 \\
\hline
\end{tabular}

para la biota, para los grupos de la tipología B modificada por expertos, y considerando los valores para los grupos de las muestras de 15 y 27 localidades de referencia. Los valores del IBMWP para los grupos de la tipología B - modificados por expertos, indican que la adición de 12 localidades de R, mejora sensiblemente la separación entre condiciones de NR y R en los grupos 2 y 8 (Tabla 3). Estas modificaciones, sin embargo, no alteran sensiblemente las puntuaciones de la mediana del IBMWP para las condiciones de referencia de cada tipo. Sólo el grupo de ríos medios bajos calcáreos, ve reducida su mediana para el IBMWP, pero se mantienen para el tipo la mediana del IHF y del número de familias (Tabla 3). Una de las mejoras que se producen es el aumento de la mediana del IASPT en algunos, indicando que la adición de nuevas localidades de referencia mejora, o no deteriora la calidad de la fauna que puede vivir en los distintos tipos de hábitat (Tabla 3).

Para cuantificar la posible contribución del IHF en la evaluación del estado ecológico de los ríos mediterráneos, se realizó un análisis de regresión logística por pasos hacia adelante (Razón de verosimilitud) (Tabla 4). Se modeló 
la probabilidad de predicción de condiciones de referencia (con 15 y 27 localidades de referencia) en función de los distintos indicadores biológicos y físico-químicos evaluados. En el modelo se incluyeron el IBMWP, IASPT, QBR, IHF, conductividad, caudal y amonio (contaminación orgánica) como variables independientes, y como variable dependiente la variable dicotómica de condición de referencia, siendo 1 cuando se cumple que es una localidad de referencia según la tipología tipo $\mathrm{B}$ - decisión de los expertos. Considerando las 15 localidades de referencia, el QBR fue la única variable predictiva incluida en el modelo con efecto positivo sobre la probabilidad de predecir la existencia de condiciones de referencia $(p<0.0001)$, con una $\mathrm{R}_{\mathrm{L}}{ }^{2}=0.35$ (Tabla 4). El análisis se realizó de nuevo, en este caso considerando las muestras de las 27 localidades de referencia, y por bloques. Un primer bloque con los descriptores biológicos (IBMWP, IASPT) y QBR, un segundo bloque con la nueva variable descriptora a analizar (IHF), y un tercer bloque con otras variables físico-químicas (amonio y conductividad). Se determinó un efecto significativo $(p<0.0001)$ y positivo de las variables IBMWP, QBR, IHF y IASPT para predecir condiciones de referencia. $L a R_{L}^{2}=0.89$ indica la mejora

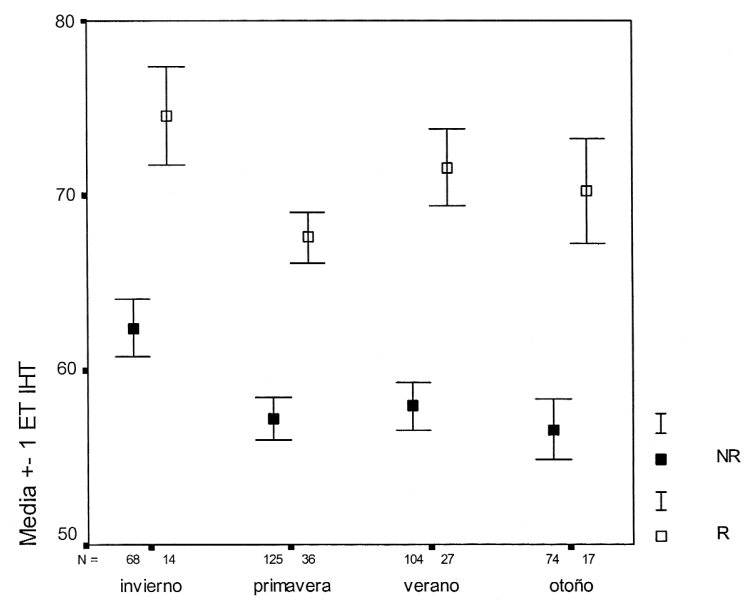

Figura 6. Variación temporal de los valores del IHF, en las localidades de referencia (27 localidades) y no referencia. Seasonal variation of IHF in 27 reference sites and in non reference sites. del ajuste del modelo, a la vez que aumenta la capacidad de pronóstico correcto de localidades de referencia al $85.3 \%$. El IHF suministra por tanto información adicional a los otros parámetros biológicos utilizados, ya que también contribuye de forma significativa a diferenciar entre condiciones de R y NR.

\section{Análisis temporal del IHF}

En la figura 6 se representa la dinámica en el tiempo de los valores del IHF, por estación y para las 27 localidades de referencia y no referencia de la matriz total del IHF de 465 muestras. Se ve como la media de las NR parece disminuir desde el invierno hacía el otoño, mientras que en las R, la tendencia es menos clara. Se realizó un análisis GLM para constatar si existía una variación temporal del hábitat, utilizando como covariables la condición de referencia y el tipo de río. Se confirmó la existencia de una dinámica temporal de sus valores a lo largo del año $(\mathrm{p}=0.049)($ Tabla 5). Esta variación temporal, se hace casi significativa para las localidades de NR (ANOVA, $F=2.59, \mathrm{p}=0.052$ ), diferenciando los valores más bajos del otoño de los del invierno más altos, y presentando la primavera y verano valores similares entre ellas, e intermedios a las otras estaciones.

Los componentes del IHF (regímenes de velocidad / profundidad, frecuencia de rápidos, diversidad de sustrato) puntúan más en invierno y otoño. $\mathrm{Y}$ en otoño, también es máximo el

Tabla 5. Análisis de la varianza (MGL) del IHF con la estación del año, utilizando como covariables la condición de referencia y el grupo de la tipología. Analyses of Variance (GLM) of IHF with season, using as covariate the reference condition and typology group.

\begin{tabular}{lrl}
\hline & & IHF \\
\hline & F & P \\
\hline Modelo corregido & 17.1 & 0.0000 \\
Estación & 2.6 & 0.0489 \\
Referencia & 66.7 & 0.0000 \\
Grupo Tipología & 12.3 & 0.0005 \\
\hline
\end{tabular}


apartado de elementos de heterogeneidad, pero la cobertura de vegetación acuática y porcentaje de sombra en el cauce son mínimos. En invierno, también el porcentaje de sombra fue máximo en las localidades de referencia, mientras que en las localidades de no referencia fue bajo todo el año. La inclusión de sedimentos fue mínima en invierno.

\section{DISCUSIÓN}

\section{Del propio índice}

El índice de hábitat fluvial diseñado en el desarrollo del proyecto GUADALMED permite evaluar la complejidad estructural del hábitat de los ríos mediterráneos, registrando la variación natural espacial existente entre tipos de ríos mediterráneos, y la dinámica temporal de los componentes del hábitat de estos sistemas. El rango de valores del IHF medido en las cuencas mediterráneas estudiadas, indica que no necesita modificarse sustancialmente la puntuación de los distintos apartados. El mínimo no puede ser 0 , ya que siempre existe el sustrato físico que puntuar, aunque este sea homogéneo (por ejemplo una rambla arenosa). Aún así no se descarta la posibilidad de introducir ligeras modificaciones y adaptaciones en el futuro.

En los ríos mediterráneos el papel estructurante de las crecidas de caudal y de los períodos secos, ocasionan la dinámica temporal de contracción y expansión del hábitat físico (Gasith \& Resh, 1999). En concordancia, La mayor relación del IHF se establece con sus componentes de frecuencia de rápidos, $\mathrm{y}$ regímenes de velocidad / profundidad, que incrementan la diversidad del hábitat en las épocas de mayor caudal de otoño-invierno, y la reducen debido al descenso de caudal en primavera y verano.

Las localidades correspondientes a tipos de ríos calcáreos bajos, medios y grandes, presentan la mayor heterogeneidad de sustrato en términos de diversidad de partícula. Probablemente por ser tramos de carácter menos erosivo y más sedimentario, donde coexisten localmente distintos tamaños de partícula, gene- rándose la máxima diversidad relativa de sustrato según Minshall (1984). Sin embargo, otros tramos fluviales presentan mayor diversidad de hábitat por tener una mayor contribución del porcentaje de sombra en el cauce y de elementos de heterogeneidad (muchos de ellos de origen alóctono), componentes que indican cierta importancia de las condiciones de heterotrofia. Estos tramos corresponden a ríos pequeños de elevada pendiente, permanentes y / o temporales, son cabeceras fluviales calcáreas y silíceas, junto con karst y ríos temporales, muchos de ellos ríos de pequeña entidad en zonas de montaña en las cuales los aportes terrestres se predice constituyen la principal fuente de energía para las comunidades acuáticas (Vannote et al., 1980; Minshall et al., 1985).

Estos resultados indican la coexistencia de diversos factores generadores de heterogeneidad ambiental a lo largo de los ríos mediterráneos; alternancia de la importancia de los elementos de heterogeneidad y cobertura de la vegetación en ríos pequeños (independientemente de su geología y temporalidad), y mayor importancia de la diversidad del sustrato en tramos bajos, medios y grandes, de ríos calcáreos. Puede ser que se concluyan similares resultados para los ríos medios y bajos silíceos, para los cuales no se han encontrado referencias en este estudio que permitan la discusión de los valores del IHF en ellos. Sin embargo, habría que tener en consideración posibles diferencias en el tamaño y forma de las partículas minerales resultantes de la transformación física a lo largo de los ríos de materiales silíceos frente a calcáreos, así como en los procesos químicos relacionados, y de influencia en la diversidad del sustrato, por ejemplo precipitación de sales y consolidación del sustrato. Así como posibles diferencias en el tipo y abundancia de la vegetación acuática, entre ambos tipos de sustratos.

Existe una disminución de la importancia de los elementos alóctonos y una reducción del tamaño medio de partícula en las llanuras aluviales y ramblas. Las ramblas constituirían el final del gradiente de disminución del tamaño de partícula mineral, de hecho muchas de ellas 
son caracterizadas como arenosas. Mientras que en las llanuras aluviales destaca la reducción de la puntuación de los elementos alóctonos y sombra en el cauce, probablemente como consecuencia de la presión de las actividades agrícolas más enfocada hacia la transformación de estas zonas. En las llanuras aluviales y ramblas se podría esperar un mayor desarrollo de vegetación acuática que compensara la disminución de los valores del índice, pero la ausencia de localidades de referencia en estos tipos en este estudio restringe la comparación.

\section{Del índice y su relación con otros parámetros biológicos}

El IHF se relaciona de forma positiva con otras variables utilizadas en la determinación del estado ecológico, implicando relaciones de interdependencia ya conocidas, pero que ayudan a entender las interrelaciones existentes entre ellas en los ríos mediterráneos. Las condiciones del hábitat son el resultado de las interrelaciones complejas entre los factores hidromorfológicos y las alteraciones antropogénicas del paisaje (Gregory et al., 1991), habiendo ejercido estas últimas una gran influencia en la zona mediterránea. La evaluación de la calidad del hábitat permite detectar impactos en la estructura del hábitat, y en último término, sobre las comunidades acuáticas (Barbour \& Stribling, 1994).

En este estudio, se han encontrado relaciones de dependencia positiva entre los valores del IHF, y el número de familias de invertebrados y su calidad biológica, con independencia del efecto de la alteración de la composición química del agua debida a la contaminación. Las relaciones entre la complejidad estructural del hábitat físico y la diversidad de especies, son un aspecto interesante a analizar en el contexto de los ríos mediterráneos estudiados. Los karst y a las cabeceras calcáreas, con una riqueza de familias media de 32.2 y 29.2 , respectivamente, y una riqueza máxima de aproximadamente $40-45$ para condiciones de referencia, presentan valores del IHF cercanos a 70. Ambos tipos de río, con similar IHF y calidad biológica media (IBMWP de aproximadamente 160) difieren en la tolerancia media de las familias que los habitan, siendo los valores más altos para las cabeceras calcáreas, de fauna claramente más reófila, frente a los karst (IASPT 5.6 frente a 4.8). Sin embargo, las cabeceras silíceas, que presentan los máximos IHF medios para condiciones de referencia de 76.8 , presentan una riqueza de familias menor (media de 23.0) que las anteriores. Es decir, la máxima diversidad del hábitat no se corresponde con la máxima riqueza de familias en los ríos silíceos, como posible explicación cabe pensar en la existencia de una fauna más diversa en sistemas calcáreos frente a silíceos en la zona mediterránea.

Ambientes más perturbados, como los ríos temporales, aunque presentan hábitats heterogéneos, están habitados por un menor número de especies (media de 18.9), frente a sistemas similares, los karsts, con hábitats parecidos, pero que constituyen sistemas acuáticos más permanentes en el tiempo (aunque dependiendo del tamaño del karst y de la longitud del periodo seco anual son temporales), y que presentan la máxima riqueza media de los ríos mediterráneos en este estudio. En este caso, y a una escala regional, la perturbación, en forma de período seco en los ríos temporales, puede ser el principal factor de regulación de la diversidad de especies (Lake, 2000).

\section{Del hábitat como predictor de condiciones de referencia.}

Los mecanismos que generan heterogeneidad y diversidad de hábitat operan a escalas espaciales locales, que comprenden desde la partícula individual, al micro hábitat, a la secuencia de rápidos y pozas (Frisell et al., 1986), a otros factores a mayor escala, como por ejemplo geología, clima, usos en la cuenca, estructura de las riberas, etc. Los estudios de colonización en ríos destacan la importancia de la regulación en el número de especies a la escala local de hábitat (Lake, 2000). Así como la importancia del mosaico de hábitats en el mantenimiento de niveles relativamente elevados de riqueza de 
especies en hábitats acuáticos naturales (Tolkamp, 1980; Pardo \& Armitage, 1997). Las variables que operan a escalas espaciales mayores, influencian la variación de las comunidades a lo largo de los ríos, y el contingente biogeográfico de especies que puede estar presente a escalas fluviales menores.

La inclusión del IHF, como factor de influencia de la calidad y riqueza biológica de las comunidades acuáticas en localidades de referencia, se demuestra válida en la práctica, y contribuye a mejorar los valores de corte estándar (como la mediana) de los parámetros biológicos. Aumentando o manteniendo la mediana de los valores del IASPT, valor que expresa la tolerancia media de las familias de invertebrados presentes en una localidad (Armitage et al., 1983).

La adición de 12 localidades de referencia en base al IHF $>66$, tampoco modifica la mediana de 75 del QBR para los grupos, el valor más bajo corresponde al grupo de ríos medianos bajos calcáreos con una mediana de 75 , y los otros grupos entre 75 y 92.5. La inclusión de otro criterio incrementa por otro lado la capacidad de pronóstico, ya que el criterio del QBR > 75, es claramente muy restrictivo y ocasiona la inclusión única del QBR como variable predictora de las condiciones de referencia, aunque el ajuste del modelo sea bajo. Además, ya existen modelos en los cuales se utilizan variables del hábitat como predictoras de la calidad biológica (Moss et al., 1987; Evans \& Norris, 1997). Por otro lado el criterio del IBMWP $>100$ es también rígido, pero no estricto, vista su variación entre los grupos de la tipología, y la amplitud de valores por encima de 100 (hasta 240 en los datos utilizados).

Los ríos mediterráneos estudiados difieren en el tamaño de las cuencas, geología de los terrenos que drenan, régimen hídrico, topografía etc. En este estudio se pone en evidencia la variación de los valores del IHF con el tipo de río, en relación a las condiciones de referencia y no referencia, y en relación con las comunidades de invertebrados que habita cada uno de estos tipos. Confirmando el establecimiento de condiciones del muy buen estado ecológico por tipo de río, y basado en el análisis de datos de localidades de referencia incluidas en este estudio, junto con otras localidades de referencia que se estudiarán en la segunda fase del proyecto GUADALMED. La propuesta de una tipología de los ríos mediterráneos y establecimiento de condiciones de referencia para los mismos, implicaría la necesidad de acotar los valores de todos los parámetros biológicos, hidromorfológicos y físico-químicos utilizados en la caracterización del estado ecológico de los ríos mediterráneos.

\section{De la variación temporal del IHF}

El valor del IHF varía estacionalmente. Esto es evidente al presentar muchos de sus componentes fluctuaciones muy contrastadas en ríos mediterráneos, por ejemplo el régimen hidrológico, estación de crecimiento de la vegetación acuática y de ribera, porcentaje de sombra en cauce, caída de hojas etc.

La dinámica estacional del IHF fue similar para localidades de referencia y no referencia, siendo más alto para las referencias en todas las estaciones del año. El hábitat de los ríos mediterráneos se ve favorecido por los mayores caudales del otoño e invierno, cuando los componentes relacionados con la hidrología producen una amplia selección de distintos tipos de sedimentos (De March, 1976), o cuando la inclusión es mínima por el lavado de sedimentos finos a mayores velocidades de corriente (Gasith \& Resh, 1999). Según los datos analizados, también la contribución de hojas, ramas, etc fue máxima en otoño, probablemente coincidiendo con la entrada de materiales con las crecidas y aumento de aportes de hojarasca en esta estación del año. En invierno se reduce la importancia de los elementos de heterogeneidad, probablemente por haber sido lavados por los altos caudales durante esta estación.

Las variables relacionadas con el caudal se ven afectadas en gran medida en los ríos mediterráneos, especialmente en los temporales, hacía el verano (frecuencia de rápidos, regímenes de velocidad / profundidad, cobertura vegetación acuática...) (Gasith \& Resh, 1999), de ahí su influencia sobre el valor general del índice en primavera y en verano. Esta reducción temporal 
de la diversidad del hábitat en sistemas temporales, se añade a la influencia temporal sobre la composición del agua por la reducción de caudal, sobre todo en localidades afectadas por enriquecimiento en nutrientes y provocando el descenso de la calidad biológica, frente a épocas de mayor dilución (Álvarez et al., 2001).

Muchas variables dependientes de las condiciones hidrológicas siguen igual dinámica en localidades de referencia y no referencia, la diversidad de sustrato, frecuencia de rápidos, regímenes de velocidad / profundidad. Sin embargo la dinámica difiere, cuando las variables son el sombreado del cauce, elementos de heterogeneidad, y cobertura de vegetación acuática, más relacionados con pulsos biológicos. Estos resultados, confirman la importancia de la evaluación del hábitat en la evaluación del estado ecológico de los ríos mediterráneos, ya que el índice incorpora por un lado la capacidad de registrar la variación temporal de la diversidad y heterogeneidad espacial del hábitat físico, así como la de registrar el impacto de las actividades humanas, de influencia más directa sobre las comunidades y procesos biológicos.

\section{AGRADECIMIENTOS}

Este trabajo se ha realizado mediante la financiación de los proyectos HID98-0323-C05 y REN2001-3438-C07 del Ministerio de Ciencia y Tecnología y PLP/10/FS/97 de la Fundación Séneca de la CARM. Nuestro especial agradecimiento al Area de Coordinación y Aplicaciones Tecnológicas de la D.G.O.H. del Ministerio de Medio Ambiente, al Àrea de Medi Ambient de la Diputació de Barcelona, a la Delegación de Granada de la Consejería de Medio Ambiente de la Junta de Andalucía y a la Agencia Catalana del Aigua por su apoyo.

\section{BIBLIOGRAFÍA}

ALBA-TERCEDOR, J. \& A. SÁNCHEZ-ORTEGA. 1988. Un método rápido y simple para evaluar la calidad biológica de las aguas corrientes basado en el de Hellawell (1978). Limnetica, 4: 51-56.

ÁlVAREZ, M., I. PARDO, G. MOYÁ, G. RAMÓN \& A. MARTÍNEZ-TABERNER. 2001. Invertebrate communities in temporary streams of the island of Majorca: a comparison of catchments with different land use. Limnetica, 20: 255-266.

ARMITAGE, P. D., D. MOSS, J. F. WRIGHT \& M. T. FURSE. 1983. The performance of a new biological Water Quality Score System based on Macroinvertebrates over a wide range of unpolluted running-water sites. Wat. Res., 17: 333-347.

BARBOUR, M. T. \& J. B. STRIBLING. 1994. A technique for assessing stream habitat structure. Proceedings of the Riparian ecosystems in the humid U.S. Function, Values and management. National Association of Conservation District, US: 156-178.

BARBOUR, M. T., J. GERRITSEN, B. D. SNYDER \& J. B. STRIBLING. 1999. Rapid Bioassessment Protocols for Use in Streams and Wadeable Rivers: Periphyton, Benthic Macroinvertebrates and Fish, $2^{\text {ond }}$ ed. EPA 841-B-99-002. US EPA, Office of Water, Washington D.C., USA.

BONADA, N., N. PRAT, A. MUNNÉ, M. PLANS, C. SOLÀ, M. ÁLVAREZ, I. PARDO, G. MOYÀ, G. RAMON, M. TORO, S. ROBLES, J. AVILÉS, M. L. SUÁREZ, M. R. VIDAL-ABARCA, A. MELLADO, J. L. MORENO, C. GUERRERO, S. VIVAS, M. ORTEGA, J. CASAS, A. SÁNCHEZORTEGA, P. JÁIMEZ-CUÉLLAR \& J. ALBATERCEDOR. 2002 - a. Intercalibración de la metodología GUADALMED. Selección de un protocolo de muestreo para la determinación del estado ecológico de los ríos mediterráneos. Limnetica, 21: 13-33.

BONADA, N., N. PRAT, A. MUNNÉ, M. RIERADEVALL, J. ALBA-TERCEDOR, M. ÁlVAREZ, J. AVILÉS, J. CASAS, P. JÁIMEZCUÉlLAR, A. MELLADO, G. MOYÁ, I. PARDO, S. ROBLES, G. RAMÓN, M. L. SUÁREZ, M. TORO, M.R. VIDAL-ABARCA, S. VIVAS \& C. ZAMORA-MUÑOZ. 2002 - b. Criterios para la selección de condiciones de referencia en los ríos mediterráneos. Resultados del proyecto GUADALMED. Limnetica, 21: 99-114

BONADA, N., N. PRAT, A. MUNNÉ, M. RIERADEVALL, J. ALBA-TERCEDOR, M. ÁlVAREZ, J. AVILÉS, J. CASAS, P. JÁIMEZCUÉLlAR, A. MELLADO, G. MOYÀ, I. PARDO, S. ROBLES, G. RAMON, M. L. 
SUÁREZ, M. TORO, M. R. VIDAL-ABARCA, S. VIVAS \& C. ZAMORA-MUÑOZ. 2002 - c. Ensayo de una tipología de las cuencas mediterráneas del proyecto GUADALMED siguiendo las directrices de la Directiva Marco del Agua. Limnetica, 21: 77-98.

BROSOFSKE, K. D., J. CHEN, R. J. NAIMAN \& J. F. FRANKLIN. 1997. Harvesting effects on microclimatic gradients from small streams to uplands in western Washington. Ecological Applications, 7: $1188-1200$.

DAVIES, P. E. 1994. National River Processes and Management Program Monitoring River Health Initiative. River Bioassessment Manual Version 1.0. Canberra: Department of Environment, Sport and Territories.

DE MARCH, B. G. E. 1976. Spatial and temporal patterns in macrobenthic stream diversity. Journal of the Fisheries Research Board of Canada, 33: 1261-70.

EVANS, L. J. \& R. N. NORRIS. 1997. Prediction of benthic invertebrate composition using microhabitat characteristics derived from stereo photography. Freshwat. Biol., 37: 621-633.

FISHER, S. G. \& G. E. LIKENS. 1973. Energy flow in Bear brook, New Hampshire: An integrative approach to stream ecosystem metabolism. Ecol. Monogr., 43: 421-439.

FOX, A. M. 1996. Macrophytes. In: River biota. Diversity and Dynamics. G. Petts \& P. Calow (eds.): 27-44. Blackwell, Oxford, USA.

FRISSELL, C. A., W .L. LISS, C. E. WARREN \& M. D. HURLEY. 1986. A hierarchical framework from stream habitat classification : viewing streams in a watershed context. Environ. Manage., 10: 199-214.

GASITH, A. \& V. H. RESH. 1999. Streams in Mediterranean climate regions: Abiotic Influences and Biotic Responses to Predictable Seasonal Events. Annu. Rev. Ecol. Syst., 30: 51-81.

GREGORY, S. V., F. J. SWANSON, W. A. McKEE \& K. W. CUMMINS. 1991. An ecosystem perspective of riparian zones. BioScience, 41, 540-551.

HYNES, H. B. N. 1970. The ecology of running waters. Toronto: University of Toronto press.

JÁIMEZ-CUÉLLAR, P., S. VIVAS, N. BONADA, S. ROBLES, A. MELLADO, M. ÁlVAREZ, J. AVILÉS, J. CASAS, M. ORTEGA, I. PARDO, N. PRAT, M. RIERADEVALL, C. E. SÁINZCANTERO, A. SÁNCHEZ-ORTEGA, M. L. SUÁREZ, M. TORO, M. R. VIDAL-ABARCA, C.
ZAMORA-MUÑOZ \& J. ALBA-TERCEDOR. 2002. Protocolo GUADALMED (PRECE). Limnetica, 21: 187-204.

LAKE, P.S. 2000. Disturbance, patchiness, and diversity in streams. J. N. Am. Benthol. Soc., 19: 573-592.

MINSHALL, G. W. 1984. Aquatic insect-substratum relationships. In: The ecology of aquatic insects. V.H. Resh \& D.M. Rosemberg (eds.): 358-400. Praeger, New York, USA.

MINSHALL, G. W., K. W. CUMMINS, R. C. PETERSEN, C. E. CUSHING, D. A. BRUNS, J. R. SEDELL \& R. L. VANNOTE. 1985. Developments in stream ecosystem theory. Can. J. Fish. Aquat. Sci., 42: 1045-1055.

MOSS, D., M. T. FURSE, J. F. WRIGHT \& P. D. ARMITAGE. 1987. The prediction of the macroinvertebrate fauna of unpolluted running-water sites in Great Britain using environmental data. Freshwat. Biol, 17: 41-52.

MUNNÉ, A, C. SOLÁ \& N. PRAT. 1998. Un índice rápido para la evaluación de la calidad de los ecosistemas de ribera. Tecnología del Agua, 175: 20-37.

NATIONAL RIVERS AUTHORITY. 1995. 1995 River Habitat Survey. Field Methodology Guidance Manual. Bristol: National Rivers Authority.

PARDO, I. \& P. D. ARMITAGE. 1997. Species assemblages as descriptors of mesohabitats. Hydrobiologia, 344: 111-128.

PARDO, I., N. FELPETO, E. S. LÓPEZ, C. FERNÁNDEZ \& C. CILLERO. 1998. Estudio de caracterización ambiental del río Louro (Pontevedra). Saneamiento general de la cuenca del rio Louro. Informe Técnico. Dirección General de Obras hidráulicas y Calidad de las Aguas. Ministerio de Obras Públicas, Transportes y Medio Ambiente.

ROBLES, S., M. TORO, C. NUÑO, J. AVILÉS, J. ALBA-TERCEDOR, M. ÁLVAREZ, N. BONADA, J. CASAS, P. JÁIMEZ-CUÉLLAR, A. MELLADO, A. MUNNÉ, I. PARDO, N. PRAT, M. L. SUÁREZ, M. R. VIDAL-ABARCA, S. VIVAS, G. MOYÁ \& G. RAMON. 2002. Descripción de las cuencas mediterráneas seleccionadas en el proyecto GUADALMED. Limnetica, 21: 35-61.

SMITH, R.L. \& T.M. SMITH. 2000. Elements of Ecology. 4th edition update. Adison Wesley Longman, Inc.

SOUTHWOOD, T.R.E. 1988. Tactics, strategies and templets. Oikos, 52: 3-18. 
SUÁREZ, M. L., M. R. VIDAL-ABARCA, M. M. SÁNCHEZ-MONTOYA, J. ALBA-TERCEDOR, M. ÁlVAREZ, J. AVILÉS, N. BONADA, J. CASAS, P. JÁIMEZ-CUÉLLAR, A. MUNNÉ, I. PARDO, N. PRAT, M. RIERADEVALL, M. J. SALINAS, M. TORO \& S. VIVAS. 2002. Las riberas de los ríos mediterráneos y su calidad: El uso del índice QBR. Limnetica, 21: 135-148.

TOLKAMP, H. H. 1980. Organism-substrate relationships in lowland streams. Agricultural Research Report 907. Agricultural University. Wageningen. The Netherlands. $211 \mathrm{pp}$.

VANNOTE, R. L., G.W. MINSHALL, K. W. CUMMINS, J. R. SEDELL \& C. E. CUSHING. 1980. The river continuum concept. Canadian Journal of Fisheries and Aquatic Sciences., 37: 130-137.

VIVAS, S., J. CASAS, I. PARDO, S. ROBLES, N. BONADA, A. MELLADO, N. PRAT, J. ALBA-
TERCEDOR, M. ÁlVAREZ, M. M. BAYO, P. JÁIMEZ-CUÉLLAR, M. L. SUÁREZ, M. TORO, M. R. VIDAL-ABARCA, C. ZAMORAMUÑOZ, N. PRAT \& G. MOYÀ. 2002. Aproximación multivariante en la exploración de la tolerancia ambiental de las familias de macroinvertebrados de los ríos mediterráneos del proyecto GUADALMED. Limnetica, 21: 149173.

VOELZ, N.J. \& J.V. MCARTHUR. 2000. An exploration of factors influencing lotic species richness. Biodiversity and Conservation, 9: 1543-1570.

WRIGHT, J.F., D. MOSS, P.D. ARMITAGE \& M.T. FURSE. 1984. A preliminary classification of running-water sites in Great Britain based on macroinvertebrate species and the prediction of community type using environmental data. Freshwat. Biol., 14: 221-256. 
Anexo 1. Estadillo de campo utilizado en la evaluación del índice de hábitat fluvial (IHF). Field form for use in the assessment of the river habitat index (IHF).

\section{Evaluación del Hábitat Fluvial para Ríos Mediterráneos. Índice IHF}

Evaluación del Hábitat Fluvial para Ríos Mediterráneos. Índice IHF
\begin{tabular}{|l|}
\hline Estación \\
\hline Operador \\
\hline
\end{tabular}

Bloques

1. Inclusión rápidos-sedimentación pozas

\begin{tabular}{l|l}
\hline Rápidos & Piedras, cantos y gravas no fijadas por sedimentos finos. Inclusión $0-30 \%$
\end{tabular}

Piedras, cantos y gravas poco fijadas por sedimentos finos. Inclusión $30-60 \%$

Sólo pozas

Piedras, cantos y gravas medianamente fijadas por sedimentos finos. Inclusión $>60 \%$.

Sedimentación $0-30 \%$

Sedimentación $30-60 \%$

Sedimentación $>60 \%$

2. Frecuencia de rápidos

Alta frecuencia de rápidos Relación distancia entre rápidos / anchura del río $<7$

Escasa frecuencia de rápidos. Relación distancia entre rápidos / anchura del río $7-15$

Ocurrencia ocasional de rápidos. Relación distancia entre rápidos / anchura del río $15-25$

Constancia de flujo laminar o rápidos someros. Relación distancia entre rápidos/anchura del río $>25$

Sólo pozas

TOTAL (una categoria)

3. Composición del substrato

\begin{tabular}{|l|l|l|l|}
\hline \multirow{2}{*}{$\%$ Bloques y piedras } & $1-10 \%$ & 2 & \\
\hline \multirow{2}{*}{$\%$ Cantos y gravas } & $>10 \%$ & 5 & \\
\hline \multirow{2}{*}{$\%$ Arena } & $1-10 \%$ & 2 & \\
\hline & $>10 \%$ & 5 & \\
\hline \multirow{2}{*}{$\%$ Limo y arcilla } & $1-10 \%$ & 2 & \\
\hline & $>10 \%$ & 5 & \\
\hline & $1-10 \%$ & 2 & \\
\hline & $>10 \%$ & 5 & \\
\hline
\end{tabular}

4. Regímenes de velocidad / profundidad

\begin{tabular}{|l|l|l|l|}
\cline { 2 - 4 } somero: $<0.5 \mathrm{~m}$ & 4 categorias. Lento-profundo, lento-somero, rápido-profundo y rápido-somero. & 10 & \\
\cline { 2 - 4 } lento: $<0.3 \mathrm{~m} / \mathrm{s}$ & Sólo 3 de las 4 categorías & 8 & \\
\cline { 2 - 4 } & Sólo 2 de las 4 & 6 & \\
\cline { 2 - 4 } & Sólo 1 de las cuatro & 4 & \\
\hline
\end{tabular}

5. Porcentaje de sombra en el cauce

\begin{tabular}{|l|l|l|}
\hline Sombreado con ventanas & 10 & \\
\hline Totalmente en sombra & 7 & \\
\hline Grandes claros & 5 & \\
\hline Expuesto & 3 & \\
\hline \multicolumn{2}{r|}{ TOTAL (una categoría) } & \\
\hline
\end{tabular}

6. Elementos heterogeneidad

\begin{tabular}{|l|l|}
\hline Hojarasca & $>10 \% \dot{\mathrm{o}}<75 \%$ \\
\cline { 2 - 2 } & $<10 \%$ ó $>75 \%$ \\
\hline Presencia de troncos y ramas \\
\hline Raíces expuestas \\
\hline Diques naturales
\end{tabular}

7. Cobertura de vegetación acuática

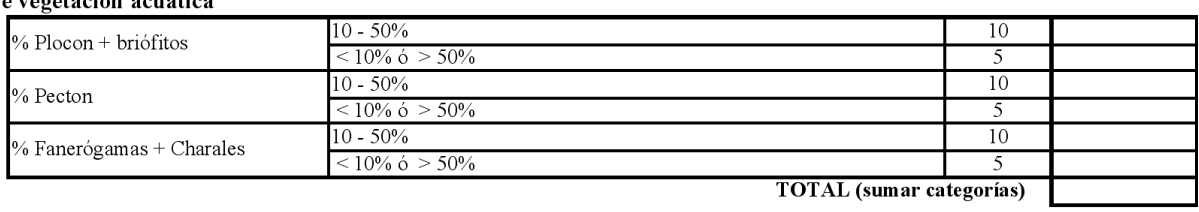

PUNTUACIÓN FINAL (suma de las puntuaciones anteriores)

La puntuación de cada uno de los apartados no puede exceder la expresada en la siguiente tabla

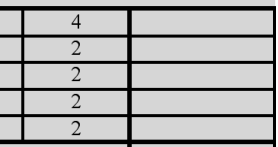

TOTAL (sumar categorias)

Inclusión rápidos - sedimentación pozas

Frecuencia de rápidos

Composición del substrato

Régimen velocidad/ profundidad

Porcentaje de sombra en el cauce

Elementos de heterogeneidad

Cobertura de vegetación acuática

2

